\title{
Desmidiaceae (Zygnematophyceae, Streptophyta) da Lagoa das Bateias, Vitória da Conquista, Bahia, Brasil
}

\author{
Maria Aparecida dos Santos ${ }^{1 *}$, Lorena Pedreira Conceição ${ }^{1}$, Fabiana Assis Pereira ${ }^{1}$, Ivania Batista de \\ Oliveira $^{2}$ \& Andrea Karla Almeida dos Santos ${ }^{1}$ \\ ${ }^{1}$ Universidade Federal da Bahia/ Instituto Multidisciplinar em Saúde-Campus Anísio Teixeira, Vitória da \\ Conquista, Bahia, Brasil. \\ ${ }^{2}$ Laboratório de Ficologia, Programa de Pós-Graduação em Botânica, Universidade Estadual de Feira de Santana, \\ Feira de Santana, Bahia, Brasil.
}

\begin{abstract}
Resumo - Este estudo objetivou identificar, descrever e ilustrar as espécies de desmídias da Lagoa das Bateias, Vitória da Conquista, estado da Bahia, baseado na análise de 15 amostras coletadas em meses das estações chuvosa (dezembro de 2010, abril e dezembro de 2011) e seca (maio e outubro de 2011). As amostras foram obtidas com auxílio de rede de plâncton com abertura de malha de $20 \mu \mathrm{m}$ e fixadas com solução de formalina a 4\%, sendo as coletas realizadas na zona litorânea da lagoa. Foram identificados e descritos 16 táxons, todos pertencentes a Desmidiaceae, representando os gêneros Cosmarium (6 espécies), Euastrum (1), Micrasterias (1), Pleurotaenium (1), Staurastrum (5) e Staurodesmus (2). Dos 16 táxons, cinco são adições à flora de algas continentais do Estado e duas variedades - Cosmarium clepsydra var. dissimile e C. tenue var. depressum - são ocorrências novas para o Brasil.
\end{abstract}

Palavras-chave adicionais: fitoplâncton, desmídias, microalgas, taxonomia.

\begin{abstract}
Desmidiaceae (Zygnematophyceae, Streptophyta) of Lagoa das Bateias, Vitória da Conquista, Bahia, Brazil) - This study aimed to identify, describe and illustrate the desmid species from the lagoon of the Bateias, Vitória da Conquista, Bahia State, Brazil, based on an analysis of 15 samples collected in months of the rainy season (December 2010, April and December 2011) and the dry season (May and of October 2011). The samples were obtained in the coastal area of the lagoon using a plankton net with mesh openings of $20 \mu \mathrm{m}$, and fixed with formalin at $4 \%$. A total of 16 taxa were identified and described, all belonging to Desmidiaceae, representing the genera Cosmarium (6 species), Euastrum (1), Micrasterias (1), Pleurotaenium (1), Staurastrum (5) and Staurodesmus (2). Amongst the 16 taxa, five are reported for the first time for the continental flora of Bahia State and two varieties - Cosmarium clepsydra var. dissimile and C. tenue var. depressum - are new reports for Brazil.
\end{abstract}

Additional key words: phytoplankton, desmids, microalgae, taxonomy.

A família Desmidiaceae agrupa organismos de hábito unicelular, isolado, de vida livre, alguns de hábito "filamentoso" unisseriado e, raramente, formas coloniais ou sem forma definida (Martins 1980; Acleto \& Zúñiga 1998; Yinxin \& Minjuan 2005). Em vista vertical, as células podem ser circulares, achatadas ou angulares (Parra \& Bicudo 1996), possuindo tipicamente uma constrição mediana denominada istmo, que divide as células em duas metades iguais, chamadas de semicélulas (Oliveira 2008). Possuem de um a muitos cloroplastídios por semicélula, os quais podem se apresentar de forma espiralada, estrelada, como placas axiais ou parietais (Oliveira 2008).

As algas conhecidas popularmente como desmídias destacam-se entre os diversos grupos de algas devido à elevada riqueza específica e complexidade da parede celular, que pode ser ornada com grânulos, verrugas, poros, espinhos e outras saliências de diferentes formas e tamanhos (Felisberto \& Rodrigues 2011). Distribuem-se de forma cosmopolita e são abundantes em ambientes de água doce, como lagos, tanques e rios oligotróficos e mesotróficos (Wehr \& Sheat 2003),

\footnotetext{
*Autora para correspondência: maria.asbio@hotmail.com

Editor responsável: Alessandro Rapini

Submetido: 30 ago. 2012; aceito: 2 jul. 2013

Publicação inicial: 7 ago. 2013; versão final: 2 maio 2014
}

podendo ser encontradas no perifíton, no metafíton e no fitoplâncton (Margalef 1983) especialmente de regiões tropicais. Dentre as famílias de Desmidiales, Desmidiaceae contribui com cerca de $70 \%$ do número total das espécies de Zygnematophyceae, incluindo gêneros com alta diversidade específica, como Cosmarium Corda ex Ralfs e Staurastrum Meyen ex Ralfs, com cerca de 1.500 e 1.200 espécies, respectivamente (Gontcharov \& Melkonian 2005).

Inicialmente, o conhecimento da ficoflórula de desmídias da Bahia contou com os seguintes estudos: Förster (1964), que identificou 59 táxons de Desmidiaceae a partir de coletas realizadas em cinco locais do estado da Bahia; Martins \& Bicudo (1987), que citaram a ocorrência de 37 táxons para o Município de Cairu; e Bicudo \& Martins (1989), com a identificação de 39 táxons de desmídias amostradas em três locais do município de Itanagra. Mais recentemente, Oliveira (2008) identificou amostras de duas áreas de proteção ambiental localizadas no município de Camaçari, documentando a ocorrência de 18 gêneros e 148 táxons, incluindo 22 novas ocorrências para o Brasil e 104 para o estado da Bahia. Esses resultados estão documentados em Oliveira et al. (2009) com 14 táxons de desmídias do gênero Micrasterias C.Agardh ex Ralfs, Oliveira et al. (2010a) com 43 táxons de Cosmarium, Oliveira et al. (2010b) 
com 15 táxons de desmídias filamentosas, Oliveira et al. (2011a,b) com os gêneros Euastrum, Xanthidium Ehrenb. ex Ralfs e Octacanthium (Hansgirg) Compère. Ehrenberg ex Ralfs, respectivamente. Finalmente, Oliveira et al. (2011c) registraram 14 novos táxons de Cosmarium para o Brasil, resultado de estudos realizados em três áreas de proteção ambiental, todas localizadas na planície costeira do estado da Bahia, e Ramos et al. (2011), estudando desmídias bromelícolas da Serra da Jiboia, documentaram 16 táxons de Desmidiaceae.

Embora as contribuições ao conhecimento das desmídias do estado da Bahia sejam crescentes, elas ainda são escassas, principalmente para o sudoeste do Estado, e considerando a alta diversidade do grupo, torna-se ainda mais necessária a publicação de estudos que visem ampliar o conhecimento da desmidioflórula na Bahia. Dessa forma, o presente estudo objetivou ampliar o conhecimento de desmídias presentes na Lagoa das Bateias, situada na cidade de Vitória da Conquista.

\section{MATERIAL E MÉtodos}

O Parque Municipal da Lagoa das Bateias (Figuras 1-4) localiza-se na zona oeste de Vitória da Conquista, entre os bairros Santa Cruz, Bateias, Loteamento Terras do Remanso, Cidade de Serrinha, Urbis II e Urbis III, compreendendo uma área de aproximadamente 53 ha, o que representa $0,4 \%$ da bacia do Riacho Santa Rita, da qual faz parte a Lagoa das Bateias (Azevedo et al. 2009). Embora o Parque tenha sido criado com o objetivo de proporcionar lazer à população, ele tem se tornado um local poluído e de odor desagradável, uma vez que grande parte do esgoto dos bairros próximos é depositada na lagoa sem tratamento.
Segundo Silveira (2011), o período chuvoso em Vitória da Conquista concentra-se nos meses de novembro a janeiro, e o período seco nos meses de maio a setembro. Assim, as coletas foram realizadas durante as estações chuvosa (dezembro de 2010, abril e dezembro de 2011) e seca (maio e outubro de 2011). As amostras foram coletadas em três pontos da zona litorânea da Lagoa das Bateias (P1- 14'51'26"S, 4052'13"W; P2- 14'51'25"S, 40 52'18"W; P3$\left.14^{\circ} 51^{\prime} 13^{\prime \prime S}, 40^{\circ} 52^{\prime} 23^{\prime \prime W}\right)$. Para a coleta do material biológico, utilizou-se rede de plâncton com abertura da malha de $20 \mu \mathrm{m}$. Após a coleta, as amostras foram armazenadas em frascos de polietileno e posteriormente fixadas com solução de formalina a $4 \%$, conforme Bicudo \& Menezes (2006) e depositados no Herbário da Universidade Estadual de Feira de Santana (HUEFS).

A medição dos espécimes foi realizada por meio de ocular micrométrica e as fotomicrografias obtidas com câmara fotográfica digital SONY, modelo Cyber-shot DSC-W7, ambos acoplados ao microscópio óptico. As identificações dos táxons foram baseadas em literatura especializada (e.g., Faustino 2006; Sophia 2009; Oliveira 2011).

\section{RESULTADOS}

Foram identificados 16 táxons de Desmidiaceae na Lagoa das Bateias, distribuídos em seis gêneros: Cosmarium (6 espécies), Euastrum (1), Micrasterias (1), Pleurotaenium (1), Staurastrum (2), Staurodesmus (5). Destes, três espécies (Staurastrum volans, $S$. mamillatus e $S$. spetsbergensis) e duas variedade (Cosmarium abbreviatum var. minus e Staurastrum furcatum var. furcatum) são novas citações para a flora de algas continentais da Bahia, e duas variedades são citadas pela primeira vez para o Brasil (Cosmarium clepsydra var. dissimile e $C$. tenue var. depressum).
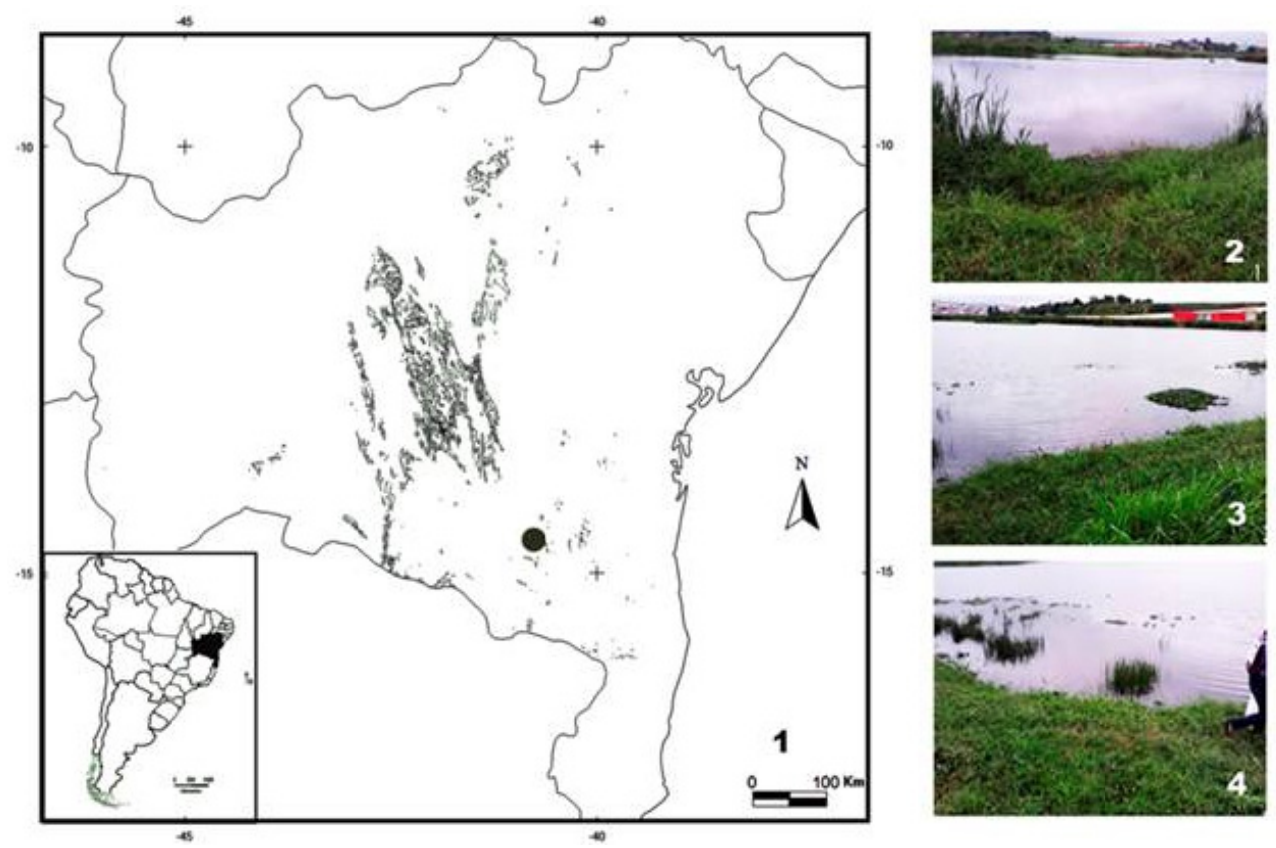

Figuras 1-4. 1- Mapa de localização do Município de Vitória da Conquista, Bahia. 2-4- Ambientes na Lagoa das Bateias. 


\section{TRATAMENTO TAXONÔMICO}

\section{Chave para os gêneros}

1. Células isoladas, compostas por duas semicélulas, que não apresentam processos ou espinhos.

2. Semicélulas de formato aproximadamente cilíndrico e alongado Pleurotaenium

2'. Semicélulas com formato variável, de piramidal a elíptica

Cosmarium

1'. Células isoladas, compostas por duas semicélulas, presença de processos e/ou espinhos.

3. Presença de fenda apical mediana ..... Euastrum 3'. Ausência de fenda apical mediana.

4. Semicélulas pentalobadas, constrição mediana profunda

Micrasterias

4'. Semicélulas diferente das descritas acima.

5. Ângulos ornados com espinho simples disposto em um só nível .. Staurodesmus

5'. Ângulos ornados com processos

Staurastrum

\section{Cosmarium Corda ex Ralfs}

1. Parede celular ornamentada por pequenos grânulos .

1'. Parede celular lisa.

C. blyttii var. blyttii

2. Células com margem apical apresentando dois dentículos nas proximidades dos ângulos

C. regnesii var. regnesii

2'. Células com margem apical sem dentículos nas proximidades dos ângulos.

3. Face da semicélula apresentando uma intumescência central

C. regnellii var. minimum

3'. Face da semicélula sem intumescência central.

4. Vista apical da célula com duas intumescências projetadas frontalmente ...

C. clepsydra var. dissimile

4'. Vista apical da célula sem intumescências projetadas frontalmente.

5. Semicélulas oblongo-trapeziformes com margem apical retusa

C. tenue var. depressum

5'. Semicélulas trapeziformes com margem apical linear a levemente côncava .. C. abbreviatum var. minus

Cosmarium abbreviatum Raciborski var. minus (W.West \& G.S.West) Krieger \& Gerloff, Die Gattung Cosmarium 3-4: 242. 1965; 2, pl. 42, fig. 18. 1969.

Figura 5.

Célula 1,1 vezes mais larga que longa, 12-14 $\mu \mathrm{m}$ larg., 11-12,5 $\mu \mathrm{m}$ compr., istmo 5,25-6 $\mu \mathrm{m}$ larg., contorno quadrangular; constrição mediana profunda, seno mediano fechado; semicélula retangular a trapeziforme, margens laterais levemente côncavas, convergente no sentido do ápice, margem apical linear a levemente côncava, ângulos arredondados; parede celular hialina a acastanhada, lisa; vistas lateral e apical oblongas; cloroplastídio axial; 1 pirenoide.

Material examinado - 14'51'25”S, 40 $52^{\circ} 18^{\prime \prime} \mathrm{W}, 20$ maio 2011, M.A. Santos et al. s.n. (HUEFS 186341); 1451'13"S, $40^{\circ} 52^{\prime} 23$ ”' 20 maio 2011, M.A. Santos et al. s.n. (HUEFS 186342).

Distribuição geográfica no Brasil. Distrito Federal (Estrela et al. 2011), Goiás (Felisberto \& Rodrigues 2004), Paraná (Felisberto \& Rodrigues 2008; 2010a,b; Biolo et al. 2013) e São Paulo (Araújo \& Bicudo 2006). Este é o primeiro registro da variedade para a Bahia.

Comentários. Cosmarium abbreviatum var. minus difere da variedade típica por apresentar medidas menores e ângulos laterais menos arredondados. Segundo Bicudo (1988), esta variedade apresenta um elevado grau de polimorfismo, o que também foi observado por Felisberto \& Rodrigues (2004) para o reservatório Corumbá, em Goiás, Felisberto \& Rodrigues (2008) para o reservatório da Usina Hidrelétrica de Salto do Vau no Paraná, e Estrela et al. (2011) em lagoas do Distrito Federal.

Cosmarium blyttii Wille var. blyttii, Forh. Vidensk. Selsk. Christiania 11: 25; pl. 1, fig. 7. 1880.

Figura 6.

Célula 1,3 vezes mais longa que larga, 17-21 $\mu \mathrm{m}$ compr., 13-19,5 $\mu \mathrm{m}$ larg., istmo 3,5-5 $\mu \mathrm{m}$ larg., contorno celular subquadrático, constrição mediana profunda, seno mediano linear, fechado; semicélula subcircular, margens laterais arredondadas e margem apical reta, crenadas, ângulos arredondados; semicélulas oblongas em vista lateral e elípticas em vista apical; parede celular hialina, com pequenos grânulos; cloroplasto axial; 1 pirenoide.

Material examinado - 14 ${ }^{\circ} 51^{\prime} 13^{\prime \prime} \mathrm{S}, 40^{\circ} 52^{\prime} 23^{\prime \prime} \mathrm{W}, 1$ abr. 2011, L.P. Conceição et al. s.n. (HUEFS 186339); 1451'25"S, 4052'18'W, 20 maio 2011, M.A. Santos et al. s.n. (HUEFS 186341).

Distribuição geográfica no Brasil. Bahia (Oliveira et al. 2010a; Ramos et al. 2011) e Paraná (Felisberto \& Rodrigues 2005; 2010b).

Comentários. Segundo Oliveira (2011), Cosmarium blyttii var. blyttii pode ser identificada pelas crenulações nas margens lateral e apical da célula e também pelos grânulos que ornamentam a face de cada semicélula.

Cosmarium clepsydra Nordst var. dissimile (Raciborski) Krieger \& Gerloff, Die Gattung Cosmarium, part 2: 145; pl. 30, fig. 9. 1965.

Figuras 7 e 8.

Célula quase tão larga quanto longa, 21,2-22,5 $\mu \mathrm{m}$ larg., 22,7-25,5 $\mu \mathrm{m}$ compr., istmo 4,5-6 $\mu \mathrm{m}$ larg., contorno celular quase circular, constrição mediana profunda, seno mediano linear, fechado; semicélulas com contorno subcircular, margens laterais arredondadas, margem apical reta; semicélulas em 
vista lateral cuneiforme, margens arredondadas, vista apical elíptica com duas intumescências projetadas frontalmente; parede celular hialina, lisa; cloroplastídio axial; 1 pirenoide.

Material examinado - 14'51'13'S, 4052'23”'W, 1 abr. 2011, L.P. Conceição et al. s.n. (HUEFS 186339); 1451'13”'S, $40^{\circ} 52^{\prime} 23^{\prime} \mathrm{W}, 20$ maio 2011, M.A. Santos et al. s.n. (HUEFS 186342)

Distribuição geográfica no Brasil. Este é o primeiro registro dessa variedade para o Brasil.

Comentários. Os exemplares da Bahia concordam com a descrição, ilustração e medidas apresentadas por Compère (1977) para o material da Bélgica. A característica mais marcante do táxon é a presença de duas intumescências arredondadas projetadas frontalmente quando a célula é observada em vista apical. A var. dissimile difere da var. bicardia (Reinsch) Croasdale, pois esta última apresenta contorno celular amplamente arredondado, bem como as margens apicais, e apresenta uma proeminência levemente marcada na região central da semicélula.

Cosmarium regnellii Wille var. minimum Eichler \& Gutw, Rozpr. Wydz. Matem.-przyr. Akad. Umiej. w Krakow. 28: 164; pl. 4, fig. 6. 1894.

Figura 9.

Célula quase tão larga quanto longa, 14-16,25 $\mu \mathrm{m}$ larg., 14-16,62 $\mu \mathrm{m}$ compr., istmo 4,3-5,5 $\mu \mathrm{m}$ larg., constrição mediana profunda, seno mediano linear, fechado; semicélula subquadrangular em vista frontal, vista apical elíptica; margens laterais lisas, retas até o meio, posteriormente convergentes para o ápice, este reto com ângulos basais subquadrangulares; faces das semicélulas apresentando uma intumescência central; parede celular hialina, lisa; cloroplastídio axial; 1 pirenoide.

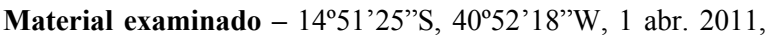
L.P. Conceição et al. s.n. (HUEFS 186338); 1451'13"S, 40²'23”'W, 1 abr. 2011, L.P. Conceição et al. s.n. (HUEFS 186339); 1451'25”S, 4052'18”'W, 20 maio 2011, M.A. Santos et al. s.n. (HUEFS 186341).

Distribuição geográfica no Brasil. Bahia (Oliveira 2011), Rio de Janeiro (Sophia 2009).

Comentários. O material analisado difere das descrições apresentadas por Oliveira (2011) e Sophia (2009), por possuir medidas celulares maiores, já que os referidos autores descreveram exemplares medindo $10-14 \times 10-12,5 \mu \mathrm{m}$ e $12-14$ x 11-14 $\mu \mathrm{m}$, respectivamente. Apesar disso, foram identificados pela presença de uma papila central na face da semicélula, característico da variedade.

Cosmarium regnesii Reinsch var. regnesii, Abh. Senckenberg Naturf. Ges. 6: 116; pl. 22, fig. A III: 1. 1876.

Figura 10.

Célula 1,3 vezes mais longa que larga, 13,5-14,5 $\mu \mathrm{m}$ compr., 10-12,5 $\mu \mathrm{m}$ larg., istmo 4-5,7 $\mu \mathrm{m}$ larg., contorno subquadrangular, constrição mediana profunda, seno mediano aberto, reto ou em forma semicircular; semicélula oblonga a sub-retangular, margem apical convexa ou levemente linear, 2 dentículos na proximidade dos ângulos, margens laterais visivelmente côncavas, margens basais levemente convexas, lisas; vista apical elíptica; parede celular hialina, projetando-se em 3 ângulos, 1 para o centro da semicélula e 2 para cada lado; cloroplastídio axial; 1 pirenoide .

Material examinado - 14'51'26”S, 4052'13”'W, 5 dez. 2010, L.P. Conceição et al. s.n. (HUEFS 186334); 1451'13"S, 40 52'23”'W, 5 dez. 2010, L.P. Conceição et al. s.n. (HUEFS 186336); 1451'25”'S, 4052'18'”, 1 abr. 2011, L.P. Conceição et al. s.n. (HUEFS 186338).

Distribuição geográfica no Brasil. Bahia (Oliveira et al. 2010a), Goiás (Nogueira 1999), Mato Grosso (Borge 1925), Minas Gerais (Lovo 1997), Paraná (Felisberto \& Rodrigues 2008, 2010b; Bortolini et al. 2010; Biolo et al. 2013).

Comentários. Medidas, descrição e ilustração da população analisada encontram-se de acordo com o observado por Oliveira et al. (2010a), para a APA Lagoas de Guarajuba e a APA Rio Capivara, Bahia, e Bortolini et al. (2010), para material do Paraná.

Cosmarium tenue Archer var. depressum IreneeMarie, Hydrobiologia 4(1/2): 134; pl. 12, fig. 11. 1952. Figura 11.

Célula 1,2 vezes mais longa que larga, 14,5-15 $\mu \mathrm{m}$ compr., 12-13,5 $\mu \mathrm{m}$ larg., istmo 9,75-11 $\mu \mathrm{m}$ larg.; constrição mediana profunda, seno mediano fechado; semicélulas oblongo-trapeziformes em vista frontal, elíptica em vista apical; margem apical retusa com ângulos arredondados; parede celular hialina lisa; cloroplastídio axial; pirenoides não observados.

Material examinado - 14'51'26”'S, 40 $52^{\circ} 13^{\prime \prime} \mathrm{W}, 20$ maio 2011, M.A. Santos et al. s.n. (HUEFS 186340); 14'51'25"S, $40^{\circ} 52^{\prime} 18^{\prime} \mathrm{W}, 20$ maio 2011, M.A. Santos et al. s.n. (HUEFS 186341).

Distribuição geográfica no Brasil. Primeiro registro da variedade para o Brasil.

Comentários. Cosmarium tenue var. depressum difere da variedade típica por apresentar seno mediano fechado, semicélulas oblongo-trapeziformes em vista frontal e elíptica em vista apical, enquanto a var. típica apresenta seno mediano aberto, semicélulas elípticas com ápices ligeiramente convexos em vista frontal e circulares em vista lateral.

\section{Euastrum Ehrenberg ex Ralfs}

Euastrum rectangulare Fritsh \& M.F.Rich, Trans. Roy. Soc. South Africa 25(2): 174; fig. 5M-N. 1937. Figuras 12 e 13.

Célula 1,2 vezes mais longa que larga, 15-16 $\mu \mathrm{m}$ compr., 12-12,75 $\mu \mathrm{m}$ larg., istmo 4-5 $\mu \mathrm{m}$ larg., contorno retangular; constrição mediana profunda, seno mediano linear; semicélula retangular, conjunto 
dos lobos basais tão largo quanto dos apicais, vista lateral retangular, vista apical elíptica; margens laterais subparalelas, 2 ondulações, 1 grânulo intramarginal em cada ondulação; ângulos basais obliquamente truncados; margem apical ondulada, 1 grânulo intramarginal em cada ondulação, ângulos apicais com um espinho pequeno; incisão apical mediana rasa, em forma de "V"; parede celular lisa; cloroplastídio axial; 1 pirenoide por semicélula.

Material examinado - 14 51 ' $26^{\prime}$ 'S, 40 $50^{\circ}$ ' $13^{\prime \prime} \mathrm{W}, 20$ maio 2011, M.A. Santos et al. s.n. (HUEFS 186340); 14 51'25”'S, 4052'18'W, 13 out. 2011, M.A. Santos et al. s.n. (HUEFS 186344); 14'51'26”'S, 4052'13”'W, 3 dez. 2011, F.A. Pereira et al. s.n. (HUEFS 186346).

Distribuição geográfica no Brasil. Amazonas (Lopes \& Bicudo 2003), Bahia (Oliveira 2011), Mato Grosso (De-Lamonica-Freire 1985), Pará (Grönblad 1945), Paraná (Biolo et al. 2008).

Comentários. Euastrum rectangulare e E. denticulatum var. rectangulare West \& G.S.West são morfologicamente próximos, e o caracter que os diferencia é a incisão apical mediana, que no primeiro apresenta-se em forma de "V" e no segundo em forma de "U”. Os espécimes analisados estão de acordo com a descrição, medidas e ilustração apresentadas por Oliveira (2011) para a APA Litoral Norte, Bahia.

\section{Micrasterias C.Agardh ex Ralfs}

Micrasterias furcata C.Agardh ex Ralfs var. furcata $\mathrm{f}$. furcata, Brit. Desmidieae: 73; pl. 9, fig. 2. 1848.

Figura 14.

Célula quase tão larga quanto longa, (130-)155$168 \mu \mathrm{m}$ larg., 150-162,5 $\mu \mathrm{m}$ compr., lobo polar (50-)68,5-72,5 $\mu \mathrm{m}$ larg., istmo 17-20(-25) $\mu \mathrm{m}$ larg., contorno subcircular, constrição mediana profunda, seno mediano fechado próximo ao istmo, aberto próximo às margens laterais da célula; semicélulas pentalobadas com depressões profundas, abertas entre os lobos, lobo polar projetado além dos lobos laterais; margens laterais com lobo subdividido em 2 lóbulos, ângulos 2-denticulados, margens basais quase retilíneas, com lobos semelhantes aos lobos laterais, dos quais se divide por uma depressão profunda, margem superior côncava com ângulos formando processos divergentes, extremidades 2-denticuladas, sendo os dentículos divergentes; parede celular hialina, lisa; vista lateral subcircular, vista apical fusiforme; cloroplastídios seguindo a forma da semicélula; vários pirenoides dispersos nas semicélulas.

Material examinado - 14 51 '13”'S, 40 52 '23”'W, 5 dez. 2010, L.P. Conceição et al. s.n. (HUEFS 186336); 1451'13”'S, 4052'23”'W, 3 dez. 2011, F.A. Pereira et al. s.n. (HUEFS 186348).

Distribuição geográfica no Brasil. Amazonas (Thomasson 1971; Förster 1974; Martins 1980; Lopes \& Bicudo 2003), Bahia (Bicudo \& Martins 1989; Oliveira et al. 2009; Oliveira 2011), Goiás (Förster 1964), Mato Grosso (Borge 1925; Souza \& Bleich
2007; Camargo et al. 2009), Minas Gerais (Nordstedt 1869; Sormus 1991; Lovo 1997), Paraná (PicelliVicentin 1984; Biolo et al. 2008; Felisberto \& Rodrigues 2011), Piauí (Förster 1964), Rio de Janeiro (Sophia \& Silva 1989) e São Paulo (Sormus \& Bicudo 1997).

Comentários. Segundo Bicudo \& Sormus (1982), Micrasterias furcata var. furcata f. furcata e M. furcata var. pseudocrux (Grönblad) C.E.M. Bicudo \& Sormus são sinônimos heterotípicos, pois a última apresenta incisões interlobares e interlobulares profundas, com o lobo polar relativamente mais estreito, de margem apical convexa e lóbulos delgados, caracteres que são típicos de $M$. furcata var. furcata f. furcata dado o amplo polimorfismo de suas populações. Os espécimes analisados estão de acordo com as medidas, descrição e ilustração apresentadas por Oliveira (2011) para o material da APA Litoral Norte, Bahia.

\section{Pleurotaenium Nägeli}

Pleurotaenium trabecula Nägeli var. trabecula f. trabecula, Gatt. Einz. Algen: 104; pl. 6, fig. A. 1849. Figura 15.

Célula 14,6 vezes mais longa que larga, 525-630 $\mu \mathrm{m}$ compr., 38-43 $\mu \mathrm{m}$ larg., istmo 20-23 $\mu \mathrm{m}$ larg., contorno aproximadamente cilíndrico, constrição mediana rasa, seno mediano aberto, ângulos arredondados; semicélula cilíndrica, margens laterais paralelas, 1 intumescência basal, 1 ou 2 ondulações leves acima da intumescência, margem apical reta, lisa, ângulos subquadráticos; parede celular hialina, pontuada; vista lateral cilíndrica, vista apical circular; cloroplastídios parietais, vários pirenoides por plastídio.

Material examinado - 14 51 '13"S, 40 52 '23"'W, 1 abr. 2011, L.P. Conceição et al. s.n. (HUEFS 186339); 1451'13"S, 40'52'23'W, 13 out. 2011, M.A. Santos et al. s.n. (HUEFS 186345); 14 51'13”'S, 4052'23”'W, 3 dez. 2011, F.A. Pereira et.al. s.n. (HUEFS 186348).

Distribuição geográfica no Brasil. Bahia (Förster 1964; Oliveira 2008, 2011), Goiás (Prescott 1957; Förster 1964), Mato Grosso (De-Lamonica-Freire 1985), Minas Gerais (Bicudo 1969), Pará (Grönblad 1945; Förster 1969; Thomasson 1971), Paraná (Cetto et al. 2004), Rio de Janeiro (Lima 1982; Sophia 1999), Rio Grande do Norte (Araújo et al. 2000) e São Paulo (Borge 1918).

Comentários. Pleurotaenium trabecula e P. ehrenbergii (Bréb.) de Bary var. ehrenbergii são morfologicamente próximas, porém a segunda apresenta um anel composto por 4 a 16 grânulos subglobosos ou pouco alongados, compondo sua ornamentação apical, o que não ocorre em P. trabecula. Os espécimes examinados apresentaram medidas que estão dentro das variações de comprimento documentadas por Förster (1964) para o estado da 
Bahia e de Goiás, e maiores que as medidas registradas por Oliveira (2011) na Bahia.

\section{Staurastrum Meyen ex Ralfs}

1. Semicélulas subglobosas em vista frontal, com presença de 2 processos ondulados, longos e bifurcados ................................................ S. volans

1'. Semicélulas elípticas em vista frontal, com presença de 2 processos retos, curtos e bifurcados

S. furcatum var. furcatum

Staurastrum furcatum (Ehrenberg) Brébisson var. furcatum, Mém. Soc. Impér. Sci. Nat. Cherbourg 4: 136, fig. 207. 1856.

Figuras 16 e 17.

Célula 1,1 vezes mais longa do que larga, 24,5-26 $\mu \mathrm{m}$ compr., 22,5-25 $\mu \mathrm{m}$ larg., istmo 9,5-12,5 $\mu \mathrm{m}$ larg., constrição mediana profunda, seno mediano aberto; margem entre os espinhos reta; semicélulas elípticas em vista lateral, vista apical 3-radiada; margem basal convexa, ângulos apicais com processos curtos divergentes, 2-denteado, 1 par intramarginal de processos curtos, 2-denteados, margem entre os ângulos côncava, margem apical quase reta; parede celular lisa, hialina; cloroplastídio parietal; muitos pirenoides.

Material examinado - 14'51'13'S, 4052'23'W, 1 abr. 2011, L.P. Conceição et al. s.n. (HUEFS 186339); 14²51'26"S, 4052'13"W, 20 maio 2011, M.A. Santos et al. s.n. (HUEFS 186340)

Distribuição geográfica no Brasil. São Paulo (Faustino 2006: Bicudo et al. 2007); este é o primeiro registro da variedade para o estado da Bahia.

Comentários. A var. típica é similar à var. aciculiferum, da qual difere por apresentar processos curtos, bifurcados, ornados por dois espinhos superpostos, e por apresentar vista celular apical triangular com dois processos bifurcados acessórios em cada ângulo (Oliveira 2011). Os espécimes analisados estão de acordo com material descrito e ilustrado por Bicudo et al. (2007) para São Paulo, exceto pelo istmo, que apresentou dimensões maiores.

Staurastrum volans W.West \& G.S.West, Trans. Linn. Soc. Lond. ser. 2 5(2): 79; pl. 9, fig. 10. 1895.

Figura 18.

Células 1,7 vezes mais largas que longas, com espinhos, 31,2-40 $\mu \mathrm{m}$ larg., 18,5-19 $\mu \mathrm{m}$ compr., istmo 6-7 $\mu \mathrm{m}$ larg., com constrição mediana profunda, seno mediano acutangular; semicélulas ovaladas ou subglobosas em vista frontal, margens basal e apical convexas; vista apical 2-radiada; ângulos apicais com processos longos divergentes, bifurcadas, apresentando 4 ou 5 ondulações; parede celular lisa; cloroplastídio axial; 1 pirenoide por semicélula.
Material examinado - 1451'26”S, 4052'13”'W, 5 dez. 2010, L.P. Conceição et al. s.n. (HUEFS 186334); 1451'13"S, 40'52'23'W, 1 abr. 2011, L.P. Conceição et al. s.n. (HUEFS 186339); 1451'26”'S, 4052'13”'W, 20 maio 2011, M.A. Santos et al. s.n. (HUEFS 186340).

Distribuição geográfica no Brasil. Acre (Lopes \& Bicudo 2003), Minas Gerais (Campos 2010), Paraná (Bortolini et al. 2008), São Paulo (Sant'Anna et al. 1989; Faustino 2006; Tucci et al. 2006; Bicudo et al. 2007) e Tocantins (Marques 2006); esse é o primeiro registro da espécie para o estado da Bahia.

Comentários. Staurastrum volans caracteriza-se por apresentar semicélulas com dois processos angulares 4- ou 5-ondulados e ausência de espinhos. Staurastrum americanum (W.West \& G.S.West) G.M.Smith é morfologicamente próxima a $S$. volans, sendo a principal diferença entre elas a vista apical da célula, que é elíptica na primeira e globosa, quase circular, na segunda. Os espécimes analisados estão de acordo com o material descrito e ilustrado por Bicudo et al. (2007) para o estado de São Paulo.

\section{Staurodesmus Teiling}

1'. Semicélulas elípticas.

2. Semicélulas portando um curto espinho inserido horizontalmente na região mediana da semicélula .................................... S. brevispina

2'. Semicélulas apresentando um curto espinho inclinado para a base da semicélula

S. lobatus var. ellipticus

1. Semicélulas oval-elípticas ou de outro formato.

3. Semicélulas torcidas e seno mediano amplamente aberto

S. tortus var. tortus

3'. Semicélulas não torcidas e seno mediano aberto em formato de ' $V$ '.

4. Semicélulas truncadamente triangulares em vista frontal .. S. mamillatus var. mamillatus

4'. Semicélulas oval-elípticas em vista frontal ... .......... S. spetsbergensis var. spetsbergensis

Staurodesmus brevispina (Brébisson) Croasdale, Trans. Amer. Microscopical Soc. 76(2): 122; pl. 3, fig. 47. 1957.

Figura 19.

Célula 1,1 vezes mais longa que larga, 28,7-32 $\mu \mathrm{m}$ compr., 25,5-27 $\mu \mathrm{m}$ larg., istmo 8,75-10 $\mu \mathrm{m}$ larg., constrição mediana profunda, seno mediano levemente aberto; semicélulas transversalmente elípticas em vista frontal, triangulares em vista apical; margens laterais e apical convexas; ângulos arredondados, ornados com um curto espinho inserido horizontalmente no terço mediano da semicélula; parede celular hialina lisa; cloroplastídio parietal; pirenoides não observados.

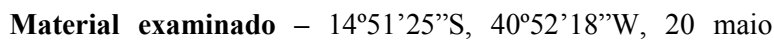
2011, M.A. Santos et al. s.n. (HUEFS 186341); 14'51'13"S, 40'52'23”'W, 20 maio 2011, M.A. Santos et al. s.n. (HUEFS 186342). 
Distribuição geográfica no Brasil. Amazonas (Bittencourt-Oliveira \& Castro 1993; Lopes \& Bicudo 2003), Bahia (Oliveira 2011), Paraná (Felisberto 2007) e São Paulo (Taniguchi et al. 2000).

Comentários. Morfologicamente, Staurodesmus brevispina é próximo de $S$. mucronatus (Ralfs) Croasd. var. paralellus (Nordst.) Teiling por apresentar semicélulas com formato elíptico e margens basal e apical convexas; entretanto, na segunda espécie, o espinho está inserido na região inferior da semicélula, enquanto em $S$. brevispina, a inserção do espinho é feita na região mediana da semicélula.
Staurodesmus lobatus (Börgesen) Bourrelly var. ellipticus (Fritsch \& Rich) Teiling, Ark. Bot. sér. 2 6(11): 586; pl. 24, fig. 6. 1967.

Figura 20.

Célula 1,4 vezes mais longa do que larga, 40-52 $\mu \mathrm{m}$ compr., 28-35 $\mu \mathrm{m}$ larg., istmo 15-20 $\mu \mathrm{m}$ larg.; constrição mediana profunda, seno mediano aberto; semicélula elíptica, margens apical e lateral côncavas, margens basais arredondadas; ângulos arredondados, ornados com um espinho hialino curto inclinado para a base da semicélula; parede celular lisa; cloroplastídio axial; pirenoides não observados.
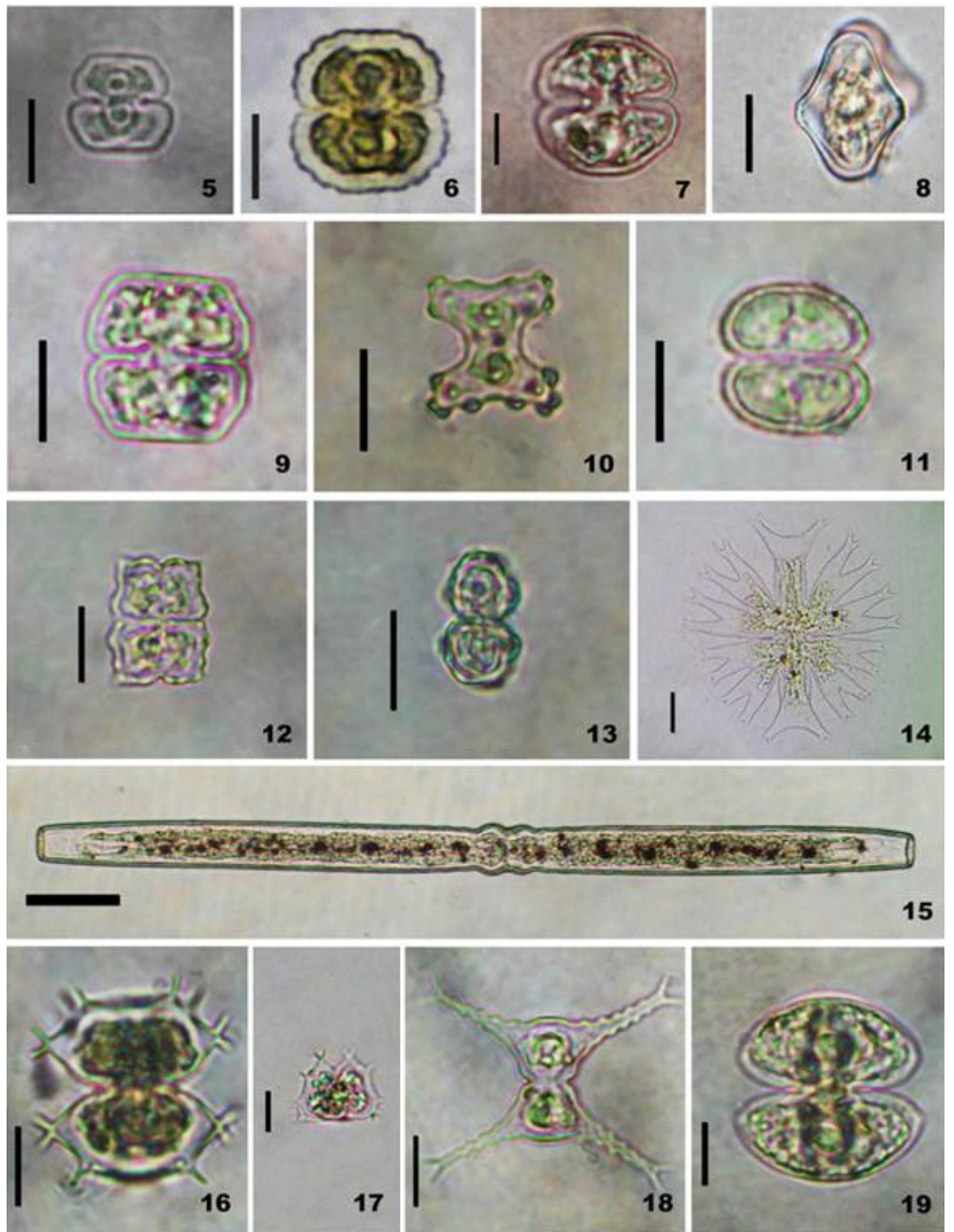

Figuras 5-19. Espécies de Desmidiaceae da Lagoa das Bateias. 5- Cosmarium abbreviatum var. minus. 6- C. blyttii var. blyttii. 7-8- C. clepsydra var. dissimile: 7- vista frontal da célula; 8- vista apical. 9- C. regnellii var. minimum. 10- C. regnesii var. regnesii. 11- C. tenue var. depressum. 12-13- Euastrum rectangulare: 12- vista frontal da célula; 13- vista lateral. 14- Micrasterias furcata var. furcata f. furcata. 15- Pleurotaenium trabecula var. trabecula f. trabecula. 16-17- Staurastrum furcatum var. furcatum: 16- vista frontal da célula; 17- vista apical. 18- Staurastrum volans. 19- Staurodesmus brevispina. (Barras: 5-13, $16-19=10 \mu \mathrm{m} ; 14=20 \mu \mathrm{m} ; 15=50 \mu \mathrm{m}$ ). 
Material examinado - 14'51'25'S, 40 $52^{\circ} 18^{\prime \prime} \mathrm{W}, 20$ maio 2011, M.A. Santos et al. s.n. (HUEFS 186341); 1451'26”'S, 4052'13”W, 13 out. 2011, M.A. Santos et al. s.n. (HUEFS 186345); 1451'25"S, 4052'18”'W, 3 dez. 2011, F.A. Pereira et al. s.n. (HUEFS 186347)

Distribuição geográfica no Brasil. Bahia (Oliveira 2011) e Pará (Gronblad 1945).

Comentários. A var. ellipticus pode ser diferenciada da típica por apresentar célula oval quando observada em vista apical (Teiling 1967). Os espécimes analisados apresentaram dimensões maiores do que o descrito e ilustrado por Oliveira (2011) para a APA Litoral Norte, Bahia.

Staurodesmus mamillatus (Nordstedt) Teiling var. mamillatus, Ark. f. Bot. 6(11): 536. 1967.

Figuras 21 e 22.

Célula 1,1 vezes mais longa que larga, 25-32,5 $\mu \mathrm{m}$ compr., 23-26 $\mu \mathrm{m}$ larg., istmo 5-6,5 $\mu \mathrm{m}$ larg., constrição mediana pouco profunda, seno mediano aberto, em forma de ' $\mathrm{V}$ '; semicélulas truncadamente triangulares em vista frontal, triangulares em vista apical; margem apical levemente côncava, com uma pequena elevação mediana, margens basais convexas; ângulos acuminados ornados com um espinho hialino de tamanho médio inclinado para a base de cada semicélula; parede celular hialina lisa; cloroplastídio parietal; pirenoides não observados.

Material examinado: $14^{\circ} 51^{\prime} 13^{\prime \prime} \mathrm{S}, 40^{\circ} 52^{\prime} 23^{\prime \prime} \mathrm{W}, 1$ abr. 2011, L.P. Conceição et al. s.n. (HUEFS 186339); 1451'26"S, 40"52'13"W, 20 maio 2011, M.A. Santos et al. s.n. (HUEFS 186340); 14'51'25'S, 4052'18'W, 13 out. 2011, M.A. Santos et al. s.n. (HUEFS 186344).

Distribuição geográfica no Brasil: Maranhão (Dellamano-Oliveira et al. 2008), São Paulo (Ferragut et al. 2005; Bicudo et al. 2007) e Paraná (Felisberto 2007; Biolo et al. 2008); este é o primeiro registro da espécie para a Bahia.

Comentários: Staurodesmus mamillatus var. mamillatus é semelhante a $S$. cuspidatus (Bréb.) Teiling, diferindo quanto a margem basal da semicélula, convexa no primeiro e retilínea no segundo. O material analisado está de acordo com descrições, ilustrações e medidas apresentadas por Bicudo et al. (2007) para material de São Paulo.

Staurodesmus spetsbergensis (Nordst.) Teiling var. spetsbergensis, Teiling, Ark. Bot. 6(1): 496; pl. 2, fig. 11. 1967.

Figuras 23 e 24.

Célula tão larga quanto longa, 29,4-30,75 $\mu \mathrm{m}$ larg., 30-31,25 $\mu \mathrm{m}$ compr., istmo 8,2-10 $\mu \mathrm{m}$ larg.; constrição mediana moderada, seno mediano aberto e em formato de ' $V$ '; semicélulas oval-elípticas em vista frontal, 3-angular em vista apical, com um curto espinho em cada ângulo; margem apical côncava, as basais convexas; ângulos arredondados ou pontiagudos, portando um espinho curto ou papila horizontal, ou inclinado para a base das semicélulas; parede celular lisa ou levemente pontoada; cloroplastídio e pirenoides não observados.

Material examinado: $14^{\circ} 51$ '26"S, 40'52' $13^{\circ}$ ' $\mathrm{W}, 20$ maio 2011, M.A. Santos et al. s.n. (HUEFS 186340); 1451'13"'S, 4052'23”W, 13 out. 2011, M.A. Santos et al. s.n. (HUEFS 186345).

Distribuição geográfica no Brasil: Rio Grande do Sul (Sophia et al. 2005); este é o primeiro registro da espécie para o estado da Bahia.

Comentários: Dentre os táxons encontrados na Lagoa das Bateias, Staurodesmus spetsbergensis var. spetsbergensis é morfologicamente semelhante a $S$. lobatus var. ellipticus quando observados em vista frontal, sendo diferenciados em relação à profundidade do seno mediano e à concavidade da margem apical da célula. Os espécimes analisados apresentaram medidas maiores do que as documentadas por Sophia et al. (2005) para material do Rio Grande do Sul.

Staurodesmus tortus (Grönblad) Teiling var. tortus, Ark. Bot. sér. B 6(11): 546; pl. 12, fig. 6-7. 1967.

Figuras 25 e 26.

Célula 1,6 vezes mais larga que longa sem espinhos, 15,5-18 $\mu \mathrm{m}$ compr., 26,2-28 $\mu \mathrm{m}$ compr. com espinhos, $15 \mu \mathrm{m}$ larg. sem espinhos, istmo $6,5-7,5 \mu \mathrm{m}$ larg.; constrição mediana moderada, seno mediano amplamente aberto; semicélulas torcidas e trapeziformes em vista frontal, 2-angular em vista apical com 1 espinho em cada ângulo; margem apical convexa, margens laterais côncavas; ângulos acuminados, contendo 1 espinho pontiagudo, curto, divergente; parede celular hialina, lisa; cloroplastídio axial; pirenoides não observados.

Material examinado: $14^{\circ} 51^{\prime} 26^{\prime \prime} \mathrm{S}, 40^{\circ} 52^{\prime} 13^{\prime \prime} \mathrm{W}, 1$ abr. 2011, L.P. Conceição et al. s.n. (HUEFS 186337); 1451'25"'S, 4052'18'W, 20 maio 2011, M.A. Santos et al. s.n. (HUEFS 186341); 14'51'25”'S, 4052'18”'W, 13 out. 2011, M.A. Santos et al. s.n. (HUEFS 186344).

Distribuição geográfica no Brasil: Bahia (Oliveira 2011), Maranhão (Dellamano-Oliveira et al. 2008), São Paulo (Bicudo et al. 2007).

Comentários: Staurodesmus tortus var. tortus é morfologicamente próxima a Tetraëdron regulare Kütz, porém a característica marcante desta última é o formato tetraédrico da célula, enquanto $S$. tortus var. tortus apresenta semicélulas torcidas e trapeziformes. Segundo Teiling (1967), essa proximidade provavelmente dificultou a identificação taxonômica da espécie, o que pode ter provocado equívocos de identificação e consequentemente mascarado a distribuição geográfica da espécie. As populações analisadas estão de acordo com as descrições, medidas e ilustrações documentadas por Oliveira (2011) para o material do Litoral Norte da Bahia. 

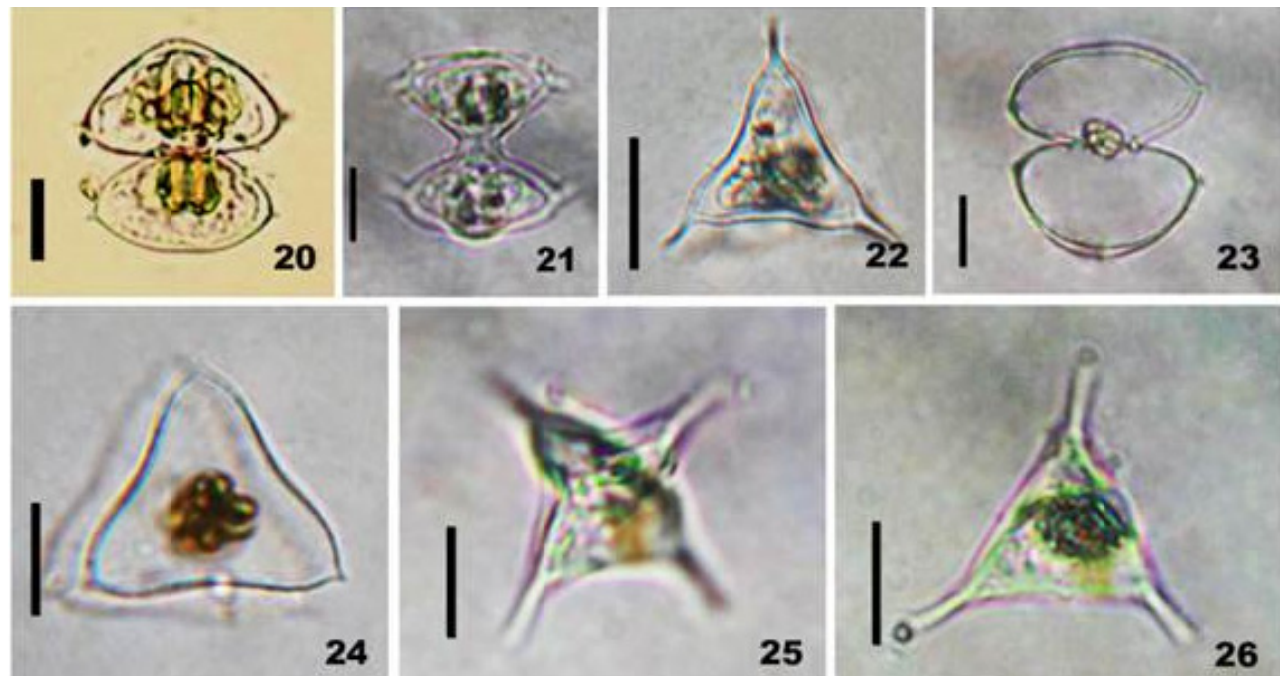

Figuras 20-26. Espécies de Staurodesmus (Desmidiaceae) da Lagoa das Bateias. 20- S. lobatus var. ellipticus. 21-22- S. mamillatus var. mamillatus: 21- vista frontal da célula; 22- vista apical. 23-24- S. spetsbergensis var. spetsbergensis: 23- vista frontal da célula; 24- vista apical. 25-26- $S$. tortus var. tortus: 25- vista frontal da célula; 26- vista apical. (Barras: $10 \mu \mathrm{m}$ ).

\section{AGRADECIMENTOS}

Ao Programa Institucional de Bolsas de Iniciação Científica da UFBA (PIBIC/UFBA), pela bolsa concedida à L.P. Conceição; ao A.F.P. Machado, pelo auxílio com a edição das imagens; aos coordenadores, funcionários e estagiários do LABZOO, LABBOT (IMS/CAT/UFBA) e do LAFICO (UEFS), pelo apoio logístico.

\section{REFERÊNCIAS}

Acleto, C. \& Zúñiga, R. 1998. Introducción a las Algas. Editorial Escuela Nueva, S.A, Lima.

Araújo, A. \& Bicudo, C.E.M. 2006. Criptógamos das Fontes do Ipiranga, São Paulo, SP. Algas 22. Zygnemaphyceae (gêneros Actinotaenium, Cosmarium e Heimancia). Hoehnea 33(2): 219237.

Araújo, M.F.F.; Costa, I.A.S. \& Chellappa, N.T. 2000 Comunidade fitoplanctônica e variáveis ambientais na Lagoa de Extremoz, Natal - RN, Brasil. Acta de Limnologia Brasileira 12: $127-140$.

Azevedo, L.P.; Santana, M.L. \& Ribas, M.C.A. 2009. Percepção ambiental na área do entorno do Parque Urbano Lagoa das Bateias, Vitória da Conquista. In: Anais do I Seminário Florestal do Sudoeste da Bahia, Vitória da Conquista, p. 147150.

Bicudo, C.E.M. 1969. Contribution to the knowledge of the desmids of the state of São Paulo, Brazil (including a few from the state of Minas Gerais). Nova Hedwigia 17(1-4): 433-549.

Bicudo, C.E.M. 1988. Polymorphism in the Desmid Cosmarium abbreviatum var. minus (Zygnemaphyceae) and its taxonomic implications. Acta Botanica Brasilica 2: 1-6.

Bicudo, C.E.M. \& Martins, D.V. 1989. Desmídias (Zygnemaphyceae) de Itanagra, estado da Bahia, Brasil. Revista Brasileira de Biologia 49(1): 309-324.

Bicudo, C.E.M. \& Menezes, M. 2006. Gêneros de Algas de Águas Continentais do Brasil: chave para identificação e descrições. 2 ed. RiMa, São Carlos.
Bicudo, C.E.M. \& Sormus, L. 1982. Desmidioflórula paulista II: gênero Micrasterias C.Agardh ex Ralfs. Bibliotheca Phycologica 57: 1-230.

Bicudo, C.E.M.; Faustino, S.M.M.; Godinho, L.R. \& Oliveira, M. 2007. Criptógamos do Parque Estadual das Fontes do Ipiranga, São Paulo, SP. Algas, 24: Zygnemaphyceae (Desmidiales: Octacanthium, Staurastrum e Staurodesmus). Hoehnea 34: 497-517.

Biolo, S.; Siqueira, N.S. \& Bueno, N.C. 2008. Desmidiaceae (exceto Cosmarium) de um tributário do Reservatório de Itaipu, Paraná, Brasil. Hoehnea 35(2): 309-326.

Biolo, S.; Bueno, N.C.; Siqueira, N.S. \& Moresco, C. 2013. New records of Cosmarium Corda ex Ralfs (Desmidiaceae, Zygnematophyceae) in a tributary of the Itaipu Reservoir, Paraná, Brazil. Acta Botanica Brasilica 27(1): 1-12.

Bittencourt-Oliveira, M.C. \& Castro, A.A.J. 1993. Ficoflórula do rio Tibagi, estado do Paraná, Brasil, II: gênero Closterium (Zygnemaphyceae). Semina, Ciências Biológicas 14: 74-85.

Borge, O. 1918. Die von Dr. A. Löfgren in São Paulo gessammelten Süsswasseralgen. Arkiv för Botanik 15(13): 1-108.

Borge, O. 1925. Die von F. C. Hoehne Wahrend der espedition Roosevelt-Rondon gessammelten Süsswasseralgen. Arkiv för Botanik 19(17): 1-56.

Bortolini, J.C.; Moresco, C.; Siqueira, N.S.; Biolo, S.; Thamis Meurer, T; Bueno, N.C. 2008. Desmidiaceae do Lago Municipal de Cascavel, Paraná, Brasil. Revista Brasileira de Biociências 6 (supl. 1): 19-21.

Bortolini, J.C.; Meurer, T. \& Bueno, N.C. 2010. Desmídias (Zygnemaphyceae) do rio São João, Parque Nacional do Iguaçu, Paraná, Brasil. Hoehnea 37(2): 293-313.

Camargo, J.C.; Loverde-Oliveira, S.M.; Sophia, M.G. \& Nogueira, F.M.B. 2009. Desmídias perifíticas da baía do Coqueiro, Pantanal Matogrossense - Brasil. Iheringia, série Botânica 64(2): 25-41.

Campos, M.O. 2010. Fatores que Influenciam a Distribuição Espacial do Fitoplâncton na Lagoa da Pampulha - BH, MG. Dissertação de Mestrado. Universidade Federal de Minas Gerais.

Cetto. J.M.; Leandrini, J.A.; Felisberto, S.A. \& Rodrigues, L. 2004. Comunidade de algas Perifíticas no reservatório de Iraí, 
Estado do Paraná, Brasil. Acta Scientiarum. Biological Sciences 1(26): $1-7$.

Compère, P. 1977. Algues de la région Du lac Tchad: VII Chlorophycophytas (3 Parte): Desmidiées (1). Hydrobiologia 11(2): 77-177.

De-Lamonica-Freire, E.M. 1985. Desmidioflórula da Estação Ecológica da Ilha de Taiamã, Município de Cáceres, Mato Grosso. Tese de Doutorado. Universidade Federal de São Paulo.

Dellamano-Oliveira, M.J.; Sant'Anna, C.L.; Taniguchi, G.M. \& Senna, P.A.C. 2008. Os gêneros Staurastrum, Staurodesmus e Xanthidium (Desmidiaceae, Zygnemaphyceae) da Lagoa do Caçó, Estado do Maranhão, Nordeste do Brasil. Hoehnea 35 $333-350$.

Estrela, L.M.B.; Fonseca, B.M. \& Bicudo, C.E.M. 2011. Desmídias perifíticas de cinco lagoas do Distrito Federal, Brasil: I - Gênero Cosmarium Corda ex Ralfs. Hoehnea 38(4): 527-552.

Faustino, S.M.M. 2006. O Gênero Staurastrum (Zygnemaphyceae) no Estado de São Paulo: levantamento florístico. Tese de Doutorado. Universidade de São Paulo - Ribeirão Preto.

Felisberto, S.A. 2007. Algas Perifiticas sobre Substrato Artificial e Natural no Rio do Corvo (Tributário do Reservatório de Rosana): composição, abundância, biomassa e produtividade. Tese de Doutorado. Universidade Estadual de Maringá.

Felisberto, S.A. \& Rodrigues, L. 2004. Periphytic desmids in Corumbá reservoir, Goiás, Brazil: genus Cosmarium Corda Brazilian Journal of Biology 64: 141-150.

Felisberto, S.A. \& Rodrigues, L. 2005. Comunidade de algas perifíticas em reservatórios de diferentes latitudes. In: L. Rodrigues, S.M. Thomaz, A.A. Agostinho \& L.C. Gomes (orgs), Biocenoses em Reservatórios: padrões espaciais e temporais. RiMa, São Carlos, p. 97-114.

Felisberto, S.A. \& Rodrigues, L. 2008. Desmidiaceae, Gonatozygaceae e Mesotaeniaceae na comunidade perifítica do reservatório de Salto do Vau (Bacia do Rio Iguaçu, PR). Hoehnea 35:235-254.

Felisberto, S.A. \& Rodrigues, L. 2010a. Periphytic algal community in artificial and natural substratum in a tributary of the Rosana reservoir (Corvo Stream, Paraná State, Brazil). Acta Scientiarum 32: 373-385.

Felisberto, S.A. \& Rodrigues, L. 2010b. Cosmarium (Desmidiaceae, Zygnemaphyceae) da ficoflórula perifítica do reservatório de Rosana, bacia do rio Paranapanema, Paraná/São Paulo, Brasil. Hoehnea 37(2): 267-292.

Felisberto, S.A. \& Rodrigues, L. 2011. Desmídias epifíticas do reservatório de Rosana, bacia do rio Paranapanema, Paraná/São Paulo, Brasil: Euastrum e Micrasterias (Desmidiaceae). Revista Brasileira de Biociencias 9(2): 206-213.

Ferragut, C.; Lopes, M.R.M.; Bicudo, D.C.; Bicudo, C.E.M. \& Vercellino, I.S. 2005. Ficoflórula perifítica e planctônica (exceto Bacillariophyceae) de um reservatório oligotrófico raso (Lago do IAG, São Paulo). Hoehnea 32(2): 137-184.

Förster, K. 1964. Desmidiaceen aus Brasilien, 2: Bahia, Goyaz, Piauhy und Nord- Brasilien. Hydrobiologia 23(3-4): 321-505.

Förster, K. 1969. Amazonische Desmidieen. 1. Areal Santarém. Amazoniana 2(1-2): 5-116.

Förster, K. 1974. Amazonische Desmidien. 2. Amazoniana 2(2): $135-242$.

Gontcharov, A.A. \& Melkonian, M. 2005. Molecular phylogeny of Staurastrum Mayen ex Ralfs and related genera (Zygnematophyceae, Streptophyta) based on coding and noncoding rDNA sequence comparisons. Journal of Phycology 41: 887-889.
Grönblad, R. 1945. De Algis brasiliensibus praecipue Desmidiaceis in regione inferiore fluminis Amazonas, a Professor August Ginzberger (Wien) ano MCMXXVII collectis. Acta Societatis Scientiarum Fennicae, sér. B, 2(6): 1-43.

Lima, M.G.S.M. 1982. Desmidiaceae (Zygnemaphyceae) do Municipio do Rio de Janeiro e Arredores: uma contribuição ao seu conhecimento. Dissertação de Mestrado. Universidade Federal do Rio de Janeiro.

Lopes, M.R.M. \& Bicudo, C.E.M. 2003. Desmidioflórula de um lago da planície de inundação do Rio Acre, Estado do Amazonas, Brasil. Acta Amazonica 33:167-212.

Lovo, I.C. 1997. Flora de Cloroficeas (Divisão Chlorophyta) de um Corpo d'Água no Campus da Universidade Federal de Viçosa, Minas Gerais, Brasil. Dissertação de Mestrado. Universidade Federal de Viçosa.

Margalef, R. 1983. Limnología. Omega, Barcelona.

Marques, A.K. 2006. Análise da Diversidade Fitoplanctônica no Reservatório da Usina Hidroelétrica Luis Eduardo Magalhães, no Médio Tocantins- TO: estrutura da comunidade, flutuações temporais e espaciais. Dissertação de Mestrado. Universidade Federal do Tocantins.

Martins, D.V. 1980. Desmidioflórula dos Lagos Cristalino e São Sebastião, Estado do Amazonas. Dissertação de Mestrado. Instituto Nacional de Pesquisas da Amazônia.

Martins, D.V. \& Bicudo, C.E.M. 1987. Desmídias da Ilha de Tinharé, estado da Bahia, Brasil. Revista Brasileira de Biologia 47(1-2): 1-16.

Nogueira, I.S. 1999. Estrutura e Dinâmica da Comunidade Fitoplanctônica da Represa Samambaia, Goiás, Brasil. Tese de Doutorado. Universidade de São Paulo.

Nordstedt, O. 1869. Desmidiaceae. Symbolae ad floram Brasiliae centralis cognoscendam, 5. Videnskabelige Meddelelser Fra Dansk Naturhistorisk Forening I Kjøbenhavn 14-15: 233-234.

Oliveira, I.B. 2008. A Família Desmidiaceae (Zygnematophyceae) Exceto Staurastrum e Staurodesmus de Duas Áreas de Proteção Ambiental, APA Rio Capivara e APA Lagoas de Guarajuba, Município de Camaçari, Bahia, Brasil. Dissertação de Mestrado. Universidade Estadual de Feira de Santana.

Oliveira, I.B. 2011. Zygnematophyceae (Streptophyta) da Área de Proteção Ambiental Litoral Norte, Bahia, Brasil. Tese de Doutorado. Universidade Estadual de Feira de Santana.

Oliveira, I.B.; Bicudo, C.E.M. \& Moura, C.W.N. 2009. Micrasterias C. Agardh ex Ralfs (Zygnematophyceae) de duas Áreas de Proteção Ambiental da planície litorânea do norte da Bahia, Brasil. Revista Brasileria de Botânica 32(2): 213-232.

Oliveira, I.B.; Bicudo, C.E.M. \& Moura, C.W.N. 2010a. Contribuição ao conhecimento de Cosmarium Corda ex Ralfs (Desmidiaceae, Zygnematophyceae) para a Bahia e o Brasil. Hoehnea 37(3): 571-600.

Oliveira, I.B., Bicudo, C.E.M. \& Moura, C.W.N. 2010b. Novas ocorrências de desmídias filamentosas (Desmidiaceae, Zygnematophyceae) para o estado da Bahia, Brasil. Acta Botanica Brasilica 24(4): 1017-1026.

Oliveira, I.B.; Bicudo, C.E.M. \& Moura, C.W.N. 2011a. Euastrum (Desmidiaceae, Zygnematophyceae) na planície litorânea do norte da Bahia, Brasil. Sitientibus série Ciências Biológicas 11(1): 62-73.

Oliveira, I.B., Bicudo, C.E.M. \& Moura, C.W.N. 2011 b. Octacanthium e Xanthidium (Desmidiaceae, Zygnematophyceae) em Camaçari, estado da Bahia, Brasil. Sitientibus série Ciências Biológicas 11(1): 89-93.

Oliveira, I.B.; Bicudo, C.E.M. \& Moura, C.W.N. 2011c. New records of Cosmarium (Desmidiaceae) to Brazil. Phytotaxa 26: $25-38$. 
Parra, O.O. \& Bicudo, C.E.M. 1996. Introduccion a la Biologia y Sistemática de las Algas de Águas Continentales. Ediciones Universidad de Concepción, Concepción.

Picelli-Vicentim, M.M. 1984. Desmídias (Zygnemaphyceae) Planctônicas do Parque Regional do Iguaçu, Estado do Paraná, Brasil: contribuição ao levantamento. Dissertação de Mestrado. Universidade Federal do Paraná.

Prescott, G.W. 1957. The Machris Brazilian expedition, Botany: Chlorophyta, Euglenophyta. Contributions in Science 11: 1-28.

Ramos, G.J.P.; Oliveira, I.B. \& Moura, C.W.N. 2011. Desmídias de ambiente fitotelmata bromelícola da Serra da Jiboia, Bahia, Brasil. Revista Brasileira de Biociências 9(1): 103-113.

Sant'Anna, C.L.; Azevedo, M.T.P. \& Sormus, L. 1989 Fitoplâncton do Lago das Garças, Parque Estadual das Fontes do Ipiranga, São Paulo, SP, Brasil: estudo taxonômico e aspectos ecológicos. Hoehnea 16: 89-131.

Silveira, P.A. 2011. Indicadores de vulnerabilidade e classificação de áreas de risco ambiental na vertente sul da Serra do Periperi em Vitória da Conquista-Bahia-Brasil. Revista Geográfica da América Central 2(47E): 1-15.

Sophia, M.G. 1999. Desmídias de ambientes fitotélmicos bromelícolas. Revista Brasileira de Biologia 59(1): 141-150.

Sophia, M.G. \& Silva, L.H.S. 1989. Considerações sobre a flora de desmídias filamentosas (Zygnemaphyceae) do noroeste de Mato Grosso e Sudeste de Rondônia, Brasil. Revista Brasileira de Biologia 49(4): 943-956.

Sophia, M.G.L. 2009. Algas Subaéreas: biodiversidade de desmídias no município de Rio de Janeiro e arredores, e estrutura de comunidades em encostas sob diferentes ações antrópicas. Dissertação de Mestrado. Universidade Federal do Rio de Janeiro.

Sophia, M.G.L.; Dias, I.C. \& Araújo, A.M. 2005. Chlorophyceae and Zygnematophyceae from the Turvo State Forest Park, state of Rio Grande do Sul, Brazil. Revista Brasileira de Biologia 51: 85-107.

Sormus, L. 1991. Desmidiaceae (Zygnemaphyceae) da Serra do Cipó, estado de Minas Gerais, Brasil, 1: Gênero Micrasterias C.Agardh ex Ralfs. Hoehnea 18(2): 1-29.

Sormus, L. \& Bicudo, C.E.M. 1997. Criptógamos do Parque Estadual das Fontes do Ipiranga, São Paulo, SP. Algas, 10: Zygnemaphyceae (Desmidiaceae, Micrasterias). Hoehnea 24(2): 75-87.

Souza, H.M.L. \& Bleich, M.E. 2007. Contribuição ao Conhecimento de Desmídias Perifíticas de uma lagoa da bacia do rio Paraguai, Mato Grosso. Revista Brasileira de Biociências 5: 132-134.

Taniguchi, G.M.; Senna, P.A.C. \& Compère, P. 2000. Desmídias (Conjugatophyceae) ocorrentes em um banco de macrófitas aquáticas da Lagoa do Diogo, 2: família Desmidiaceae (tribos Euastreae, Xanthideae, Staurastreae e Hyalotheceae). In: J.E. Santos \& J.S.R. Pires (eds), Estação Ecológica de Jatai: estudos integrados em ecossistemas. Vol. 2. RiMa, São Carlos, p. $347-867$.

Teiling, E. 1967. The desmid genus Staurodesmus: a taxonomic study. Arkiv for Botanik, ser. 2, 6(11): 467-629.

Thomasson, K. 1971. Amazonian desmids. Mémoires Institute Royal des Sciences Naturelles de Belgique, ser. 2, 86: 1-57.

Tucci, A.; Sant'Anna, C.L.; Gentil, R.C. \& Azevedo, M.T.P. 2006. Fitoplâncton do Lago das Garças, São Paulo, Brasil: um reservatório urbano eutrófico. Hoehnea 33(2): 147-175.

Wehr, J.D. \& Sheat, R.G. 2003. Freshwater Algae of North America. Ecology and classification. Academic Press, New York.

Yinxin, W. \& Minjuan, Y. 2005. Phytoplanktonic desmids community in Donghu Lake, Wuhan, China. Chinese Journal of Oceanology and Limnology 23(1): 519-525. 